\title{
ON THE LATTICE OF CONGRUENCES ON A SEMILATTICE
}

\author{
T. E. HALL ${ }^{1}$ \\ (Received 9 May 1969) \\ Communicated by G. B. Preston
}

\section{Introduction and summary}

Lattices of congruences are studied in section II.6 of Cohn [2]. Papers [5] and [6] by Munn deal with lattices of congruences on semigroups and conditions under which these lattices are modular. In [4] Lallement shows that the lattice of congruences on a completely 0 -simple semigroup is semimodular, giving an alternative proof of the result, due to Preston [7], that the lattice of congruences on a completely 0 -simple semigroup satisfies a certain chain condition which is a natural extension to arbitrary lattices of the Jordan-Dedekind chain condition for finite lattices.

In this paper we show that this chain condition is also satisfied by the lattice of congruences on any semilattice, and we show that this lattice is upper-semimodular, and in fact satisfies the condition (1) if $y>x \wedge y$ then $x \vee y \succ x$, for any lattice elements $x$ and $y$. (For any elements $a, b \in L, a>b$ means that $a \geqq b$ and that $a \neq b$, and $a \succ b$ ( $a$ covers $b$ ) means that $a>b$ and there is no element $x \in L$ such that $a>x>b$. For a lattice $L$ with a zero element 0 , the atoms of $L$ are those elements which cover 0 .) The proofs are easy once we determine the atoms in the lattice of congruences on any semilattice.

\section{Preliminaries}

Result 1. (This is easy to show and is largely contained in section 3 of [8].) Let $S$ be any set and consider the set of equivalence relations on $S$, ordered under set-inclusion. Let $\sigma$ be any equivalence on $S$. Then there is a natural order-preserving one-to-one correspondence (described below) between the set of equivalences on $S$ which contain $\sigma$ and the set of all equivalences on the set $S / \sigma$. For any equivalence $\gamma$ on $S$ containing $\sigma$, the corresponding equivalence on $S / \sigma$ is

$$
\gamma / \sigma=\{(x \sigma, y \sigma) \in S / \sigma \times S / \sigma:(x, y) \in \gamma\} .
$$

1 This research was carried out at Monash University while the author held a Commonwealth Postgraduate Award. 
If $S$ is a semigroup and $\sigma$ is a congruence on $S$ then an equivalence $\gamma$ on $S$ containing $\sigma$ is a congruence on $S$ if and only if $\gamma / \sigma$ is a congruence on the semigroup $S / \sigma$.

The following definitions and results are contained in [3], except for the definition of upper-semimodularity, which is merely called condition (7) in [3].

Definttion 1. (From definition 1, page 85 [3].) A lattice L is semimodular when for any elements $x, y, z \in L$ if

$$
y \wedge z<x<z<x \vee y
$$

then there exists an element $t \in L$ such that

$$
y \wedge z<t \leqq y \text { and } x=(x \vee t) \wedge z
$$

DEFINITION 2. A lattice $L$ is upper-semimodular when for any elements $x, y \in L$, if

$$
x \succ x \wedge y \text { and } y \succ x \wedge y \text { then } x \vee y \succ x \text { and } x \vee y \succ y .
$$

Result 2. (From lemma 1 and theorem 1, p. 265 [3].) The lattice $\mathscr{E}(S)$ of all equivalences on a set $S$ is semimodular.

Result 3. (From property 2, page 90 [3].) Any semimodular lattice satisfies the condition (1) of section 1 .

Result 4. (From page 92 [3].) Any lattice satisfying condition (1) is uppersemimodular.

RESULT 5. (From theorem 1, page 88 [3].) Let $L$ be any lattice satisfying condition (3), and let $a, b$ be elements of $L$ such that $a<b$. If there is a maximal chain of elements from $a$ to $b$ which is of finite length, then any maximal chain of elements from $a$ to $b$ is finite and of the same length.

We shall use whenever possible, and often without comment, the notations and conventions of Clifford and Preston [1].

\section{On $\Lambda(Y)$}

Let $Y$ be any semilattice and let $\Lambda(Y)$ be the lattice of congruences on $Y$.

THEOREM 1. Let $\rho$ be any atom of $\Lambda(Y)$. Then there exist elements $e, f \in Y$ such that

(i) $\rho=\{(e, f),(f, e)\} \cup \imath_{Y}$ (where $t_{Y}$ denotes the identity relation on $Y$.)

(ii) $f<e$, and for any element $x \in Y$, if $x<e$ then $x \leqq f$.

Conversely, for any pair $e, f \in Y$ such that (ii) holds, the relation $\rho$ defined by (i) is a congruence on $Y$ and hence is an atom of $\Lambda(Y)$. 
Condition (ii) (clearly) implies that $f \prec e$ and is (clearly) equivalent to the condition that $\mathrm{e} Y=f Y \cup\{e\}$.

Proof. Let $\rho$ be any atom of $\Lambda(Y)$. Then there exists a pair $(e, f) \in \rho$ such that $e \neq f$, whence either $e \neq e f$ or $e f \neq f$. We may assume without loss of generality that $e \neq e f$ (since $(f, e) \in \rho$ and $e f=f e$ ). Then $e f<e$ and $(e, e f)=$ $(e e, e f) \in \rho$.

For each ideal $I$ of $Y$ define the relation $\sigma_{I}=(I \times I) \cup l_{Y}$, a congruence on $Y$. Then $\rho \cap \sigma_{e Y}$ is a congruence on $Y$ such that

$$
l_{Y} \subset \rho \cap \sigma_{e Y} \subseteq \rho,
$$

since $(e, e f) \in \rho \cap \sigma_{e Y}$, and so

$$
\rho=\rho \cap \sigma_{e Y} .
$$

Since $(e, f) \in \rho=\rho \cap \sigma_{e Y}$, we have $(e, f) \in(e Y) \times(e Y)$, so $f \in e Y$ and $e f=f<e$.

Take now any element $x \in Y$ such that $x<e$. Then $(e, f) \notin \sigma_{x Y}$ and so

whence

$$
\imath_{Y} \subset \rho \cap \sigma_{x Y} \subseteq \rho,
$$

$$
\rho \cap \sigma_{x Y}=\imath_{Y} \text { whenever } x<e .
$$

Moreover

$$
(x, x f)=(x e, x f) \in \rho \cap \sigma_{x Y}=\imath_{Y}
$$

whence $x=x f$, i.e. $x \leqq f$. Hence condition (ii) is satisfied for $e$ and $f$.

Take now any pair $(x, y) \in \rho$ with $x \neq y$. From (2), $x, y \in e Y$. Suppose, for the moment that $x<e$ and $y<e$; then $x, y \leqq f$ and, by (3),

$$
(x, y) \in \rho \cap \sigma_{f Y}=l_{Y}
$$

whence $x=y$, a contradiction.

Suppose that $x=e$. Then $y<e$ since $y \in e Y$ and $y \neq x=e$, and so $y \leqq f$. From $(e, f) \in \rho$ and $(e, y)=(x, y) \in \rho$ we obtain

$$
(f, y) \in \rho \cap \sigma_{f Y}=l_{Y},
$$

and so $y=f$. Similarly, if we suppose $y=e$ then we can prove that $x=f$. It follows that $\rho=\{(e, f),(f, e)\} \cup \imath_{Y}$, giving (i).

Conversely, suppose that $e, f$ are any elements of $Y$ for which (ii) holds, and define $\rho$ by (i). Take any element $g \in Y$. Then if $e g=e$, we have

$$
(e g, f g)=(e g,(f e) g)=(e, f e)=(e, f) \in \rho
$$

and if $e g<e$, we have $e g \leqq f$ and so

$$
(e g, f g)=(e g, f e g)=(e g, e g) \in \rho .
$$

It follows that $\rho$ is a congruence. 
THEOREM 2. For any two congruences $\rho$ and $\sigma$ on $Y, \rho>\sigma$ in the lattice $\Lambda(Y)$ if and only if $\rho \succ \sigma$ in the lattice $\mathscr{E}(Y)$.

Proof. The 'if' statement is obvious.

Suppose that $\rho \succ \sigma$ in the lattice $\Lambda(Y)$. Then $\rho / \sigma \in \Lambda(Y / \sigma)$ (by result 1 ) and since the one-to-one correspondence of result 1 is order-preserving, $\rho / \sigma$ is an atom of $\Lambda(Y / \sigma)$. But $Y / \sigma$ is also a semilattice, whence by theorem $1, \rho / \sigma$ is an atom of $\mathscr{E}(Y / \sigma)$. Using result 1 again we obtain that $\rho \succ \sigma$ in the lattice $\mathscr{E}(Y)$.

Theorem 3. The lattice $\Lambda(Y)$ satisfies condition (1).

Proof. From corollary 6.5, page $88[2] \Lambda(Y)$ is a sublattice of $\mathscr{E}(Y)$, and $\mathscr{E}(Y)$ satisfies condition (1) by results 2 and 3. Theorem 3 now follows from theorem 2.

COROLLARY 1. If $\rho$ and $\sigma$ are two congruences on $Y$ such that $\sigma \subseteq \rho$ and there is a maximal chain of congruences from $\sigma$ to $\rho$ which is of finite length, then any maximal chain of congruences from $\sigma$ to $\rho$ is finite and of the same length.

COROLlaRY 2. The lattice $\Lambda(Y)$ of congruences on a semilattice $Y$ is uppersemimodular.

Example 1. This example is due to J. M. Howie and shows that the results following theorem 1 do not extend to semilattices of groups (and hence inverse semigroups). Put $S=\{e, a, f, b\}$ with multiplication given by the table

\begin{tabular}{c|cccc}
- & $e$ & $a$ & $f$ & $b$ \\
\hline$e$ & $e$ & $a$ & $f$ & $b$ \\
$a$ & $a$ & $e$ & $b$ & $f$ \\
$f$ & $f$ & $b$ & $f$ & $b$ \\
$b$ & $b$ & $f$ & $b$ & $f$
\end{tabular}

Theorem 4.11 [1] can be used to show easily that $S$ is a semigroup which is a semilattice of groups. The lattice of all congruences on $S$ is isomorphic to the lattice given by the following diagram

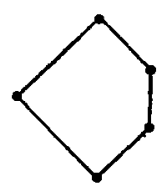

This lattice is the smallest lattice which is not upper semimodular, in bcth of the following senses: it is isomorphic to a sublattice of any lattice which is not upper semimodular; (whence) it is the lattice of least order which is not upper semimodular. 


\section{References}

[1] A. H. Clifford and G. B. Preston, The Algebraic Theory of Semigroups, Vol. I (Math. Surveys, number 7, Amer. Math. Soc., 1961).

[2] P. M. Cohn, Universal Algebra (Harper and Row, New York, 1965).

[3] M. L. Dubreil-Jacotin, L. Lesieur and R. Croisot, Leçons sur la théorie des treillis des structures algébriques ordonnées et des treillis géométriques (Éditeur-imprimeur-libraire, GauthierVillars, Paris, 1953).

[4] G. Lallement, 'Demi-groupes reguliers', (Doctoral dissertation) Annali di Matematica pura ed applicata 77 (1967), 47-130.

[5] W. D. Munn, 'A certain sublattice of the lattice of congruences on a regular semigroup', Proc. Camb. Phil. Soc. 60 (1964), 385-391.

[6] W. D. Munn, 'The lattice of congruences on a bisimple $\omega$-semigroup'. Proc. Roy. Soc. Edinburgh, 67 (1966), 175-184.

[7] G. B. Preston, 'Chains of congruences on a completely O-simple semigroup', J. Aust. Math. Soc. 5 (1965), 76-82.

[8] N. R. Reilly and H. E. Scheiblich, 'Congruences on regular semigroups', Pacific J. Math. 23 (1967), 349-360.

\section{Monash University}

Clayton 